\title{
What's new in functional urology research?
}

\author{
Sender Herschorn, MD, FRCSC
}

Professor, Division of Urology, University of Toronto, Sunnybrook Health Sciences Centre, Toronto, ON, Canada

Cite as: Can Urol Assoc J 2016;10(5-6Supp|2):S124-6. http://dx.doi.org/10.5489/cuaj.3913

\section{The addition of mirabegron to solifenacin for incontinent overactive bladder $(O A B)$ results in an acceptable cardiovascular profile}

Oral pharmacotherapies for the treatment of $O A B$ include antimuscarinics such as solifenacin and the beta- 3 adrenoceptor ( $\beta 3 \mathrm{AR}$ ) agonist mirabegron. Although both classes of drugs are similarly effective, the antimuscarinics have been associated with bothersome anticholinergic side effects, such as dry mouth and constipation, that can lead to treatment discontinuation. The randomized, double-blind, multicentre, phase 3 BESIDE study showed that add-on therapy with mirabegron $50 \mathrm{mg}$ for 12 weeks provided additional improvements in $\mathrm{OAB}$ symptoms for incontinent $\mathrm{OAB}$ patients who experience an insufficient response to solifenacin $5 \mathrm{mg}$ compared with solifenacin $5 \mathrm{mg}$ or $10 \mathrm{mg}$ monotherapy. ${ }^{1}$ Since antimuscarinics and $\beta 3 A R$ agonists are both potentially autonomically active, the BESIDE investigators examined cardiovascular outcomes in this study population, and reported these results at EAU 2016. ${ }^{2}$ Key parameters of blood pressure and pulse rate were assessed at screening, baseline, and intervals throughout the study. Electrocardiogram (ECG) results were also assessed at screening, baseline, and end of study. No clinically meaningful differences in blood pressure or heart rate were observed between the treatment groups, and there were no significant changes in ECG properties. These results provide some assurance that the addition of mirabegron to solifenacin in patients with incontinent $O A B$ who have an inadequate response to solifenacin does not increase risk of cardiovascular adverse events.

\section{$\beta 3 A$ Rs may be expressed in sensory nerves}

The $\beta 3 \mathrm{AR}$ agonist mirabegron has long been thought to ameliorate symptoms of $O A B$, such as urinary urgency or urgency incontinence, by targeting the $\beta 3 A R$ and causing detrusor muscle relaxation. However, it has been suggested that these therapies may also reduce activity in sensory nerves. Immunohistochemistry of cadaveric organs was conducted by a group from Portugal and the U.K., showing that immunoreactivity for $\beta 3 A R$ was present throughout the human bladder in both the mucosa in nerve fibres intermingled on the connective tissue and in the muscular layer, on nerve fibres present along smooth muscle bundles. ${ }^{3}$ Surprisingly, no immunoreaction was detected in smooth muscle cells, suggesting that this is not the therapeutic target for $\beta 3 A R$ agonists. The hypothesis that the therapeutic effects of $\beta 3 A R$ agonists actually occur through modulation of the bladder neural circuits rather than a direct effect on smooth muscle cells warrants further exploration.

\section{Botulinum toxin type A effective in decreasing urgency urinary incontinence in patients with idiopathic $O A B$}

Studies comparing the effectiveness of botulinum toxin type A and anticholinergics for the treatment of urgency urinary incontinence are lacking. Everaert, on behalf of colleagues from other countries, presented results of a prospective, multicentre, randomized, controlled trial evaluating the efficacy and safety of botulinum toxin type $\mathrm{A} 100 \mathrm{U}$ or solifenacin $5 / 10 \mathrm{mg}$ vs. placebo in 356 solifenacin-naive patients with idiopathic $\mathrm{OAB}$ and urinary incontinence who were inadequately managed by one or more anticholinergic agent. ${ }^{4}$ At study entry, patients reported an average of 4.94 urge incontinence episodes (UIE)/day at baseline. After 12 weeks of treatment, daily UIE were significantly reduced by both botulinum toxin type A and solifenacin; however, these reductions were significantly greater with botulinum toxin type A (Table 1). The proportions of patients who achieved $100 \%, 75 \%$ or higher, and $50 \%$ or higher reductions in daily UIE were also higher among botulinum- and solifenacintreated patients than placebo-treated patients. Again, these rates were higher among botulinum-treated patients than solifenacin-treated patients - significantly so for reductions of $50 \%$ or higher and $75 \%$ or higher. No new safety signals were observed during the study. 
Table 1. Change from baseline in urinary incontinent episodes (UIE)/day and proportions of patients achieving $100 \%, 75 \%$ or greater, and $50 \%$ or greater reductions in UIE $^{4}$

\begin{tabular}{|c|c|c|c|}
\hline Parameter, n (\%) & $\begin{array}{c}\text { Botulinum } \\
\text { toxin type } \\
\text { A } 100 \mathrm{U} \\
(\mathrm{n}=145)\end{array}$ & $\begin{array}{l}\text { Solifenacin } \\
5 / 10 \mathrm{mg} \\
(\mathrm{n}=151)\end{array}$ & $\begin{array}{c}\text { Placebo } \\
(n=61)\end{array}$ \\
\hline $\begin{array}{l}\text { LS mean change from } \\
\text { baseline in UIE/day }\end{array}$ & $-3.19^{a, b}$ & $-2.56^{a}$ & -1.33 \\
\hline $\begin{array}{l}\text { Proportion of patients } \\
\text { achieving } 100 \% \text { UIE reduction }\end{array}$ & $33.8 \% \%^{a, c}$ & $24.5 \%^{\mathrm{d}}$ & $11.7 \%$ \\
\hline $\begin{array}{l}\text { Proportion of patients } \\
\text { achieving } \geq 75 \% \text { UIE reduction }\end{array}$ & $55.2 \%^{a, e}$ & $43.0 \%^{f}$ & $21.7 \%$ \\
\hline $\begin{array}{l}\text { Proportion of patients } \\
\text { achieving } \geq 50 \% \text { UIE reduction }\end{array}$ & $77.2 \%{ }^{\mathrm{a}, \mathrm{g}}$ & $64.9 \%{ }^{a}$ & $33.3 \%$ \\
\hline
\end{tabular}

\section{Mirabegron improves nocturia and nocturia-associated quality of life in affected women}

Nocturia can negatively impact quality of sleep, mood, and overall health. However, limited options are available to treat this common, bothersome symptom. Yoshida and colleagues from Japan presented results of their prospective, multicentre study evaluating the effects of mirabegron $50 \mathrm{mg} /$ day on nocturia, nocturia-associated quality of life (QOL) and sleep quality in 60 female patients with $\mathrm{OAB}$ and nocturia. ${ }^{5}$ After 12 weeks of treatment, mirabegron significantly improved total International Prostate Symptom Score (IPSS), QOL index, voiding symptoms and storage symptoms, as well as nocturnal frequency, nocturnal urine volume per void, urine volume of the first nocturnal voiding and nocturia-QOL scores. Mirabegron also increased hours of undisturbed sleep, from 160.6 to 203.8 minutes. In addition, Pittsburgh Sleep Quality Index scores were improved significantly after treatment. These preliminary data suggest that mirabegron should be evaluated further to explore its effects on nocturia and QOL.

\section{The addition of desmopressin to alpha-blocker therapy is effective and well-tolerated in men with persistent nocturia}

Alpha-blockers have been used to treat symptoms of nocturia in men, resulting in measureable, albeit modest, improvements in symptoms related to bladder outlet obstruction. Recently, there has been increased interest in the use of desmopressin, a synthetic analogue of arginine vasopressin, to treat this bothersome condition. A group of researchers from Korea investigated the efficacy and safety of desmopressin add-on therapy for men with persistent nocturia despite treatment with an alpha-blocker for lower urinary tract symptoms (LUTS). ${ }^{6}$ This placebo-controlled study enrolled men 40-65 years of age with LUTS who had been taking an alpha-blocker for a minimum of eight weeks and who were experiencing nocturia two or more times per night. The men were randomly assigned to treatment with either desmopressin 0.2 mg add-on therapy $(n=47)$ or placebo $(n=39)$. Men who received desmopressin add-on therapy experienced significantly greater reductions in nocturnal urine volume $(-283.6$ vs. $-112.6 ; \mathrm{p}<0.001)$, Nocturnal Polyuria Index (NPI) $(-13.9$ vs. $5.9 ; p=0.001)$, IPSS total score (-5.2 vs. $-2.5 ; p=0.042)$, IPSS Nocturia score (-1.4 vs. $-0.6 ; \mathrm{p}=0.004)$, and International Consultation on Incontinence Questionnaire-Nocturia $(\mathrm{IClQ}-\mathrm{N})$ score $(-3.3$ vs. $-1.6 ; \mathrm{p}=0.001)$. There were no significant differences in adverse events between the two groups. Interestingly, there were no significant differences between the two groups with respect to patients' perceptions of treatments, with similar proportions perceiving "much benefit" or feeling "very satisfied" with the treatment. However, significantly more patients treated with desmopressin add-on therapy were "very willing to continue treatment" $(82.9 \%$ vs. $61.5 \% ; p=0.025)$, suggesting that patients tolerated the combination therapy well.

\section{Prostatic urethral lift offers rapid, durable improvements in LUTS with minimal adverse effects}

The LIFT study was a prospective, randomized study comparing prostatic urethral lift $(n=140)$ with sham procedure $(n=66)$ in men with symptomatic LUTS in 19 centres across the U.S., Canada, and Australia. At three months, the urethral lift outperformed sham procedure, with an $88 \%$ reduction in IPSS compared with sham $(p=0.003) .^{7}$ Medium- to long-term outcomes from the LIFT study were presented at EAU 2016, ${ }^{8}$ and showed that this response remained relatively persistent throughout four years of followup. After four years, IPSS remained improved by $41 \%$ compared with baseline, peak flow remained improved by $62 \%$ compared with baseline, and $86.4 \%$ of patients still did not require additional treatment for their LUTS symptoms. This highquality, randomized study highlights several advantages of the prostatic urethral lift, including rapid, durable symptom relief and preservation of sexual function. Durability will continue to be assessed through protocol-driven followup through to five years.

\section{Urodynamics remain an important tool for identifying bladder outlet obstruction}

Voiding function is often defined by the bladder outlet obstruction index (BOOI) and bladder contractility index $(\mathrm{BCI})$. To establish whether there is a relationship between free flow (FF) at the maximum flow rate and these two indices, a group from the Bristol Urological Institute in the U.K. retrospectively analyzed 1717 men who underwent both FF 
and pressure/flow studies in their institution between 2010 and 2015, with similar voided volumes recorded for both measures. ${ }^{9}$ Among men with a FF of less than $10 \mathrm{~mL} / \mathrm{s}$, $60 \%$ were obstructed; however, surprisingly, $15 \%$ were not obstructed. Conversely, $65 \%$ of men with a FF of more than $15 \mathrm{~mL} / \mathrm{s}$ were unobstructed as expected, but $15 \%$ were obstructed. There was no association between $\mathrm{FF}$ and $\mathrm{BCl}$, but the majority of men were categorized as having normal $\mathrm{BCl}$. These results show that, regardless of the FF cut-point, there are a significant number of false negatives and false positives for obstruction, underscoring the importance of considering invasive urodynamics before embarking on prostate de-obstructing surgery.

\section{Adverse life events are associated with depression in men with LUTS}

LUTS and depression commonly coexist in elderly men, negatively impacting QOL. Using data from the multicentre, population-based European Male Ageing Study, researchers sought to further clarify the nature of this relationship by evaluating adverse life events (ALEs) as potential moderators and the inflammatory reaction as an underlying biological mechanism. ${ }^{10}$ Gacci and colleagues reported their results from 3369 men who were assessed by means of several self-reported questionnaires, including the IPSS, the Beck's Depression Inventory-II (BDI-II), and the Adverse Life Events Scale. Information was also collected about their general health, lifestyle, and medical comorbidities. Biological markers, including prostate-specific antigen (PSA), testosterone, and $\mathrm{C}$-reactive protein were recorded.

BDI scores were strongly associated with the IPSS total score $\left(R^{2} 0.44 ; \beta=0.13 ; p<0.001\right)$, as well as with the IPSS voiding score (Spearman rho $0.26 ; p<0.001$ ) and storage (Spearman rho 0.25; $\mathrm{p}<0.001$ ) subscales. Moreover, having at least one ALE was associated with a higher risk of developing LUTS compared with having no ALE (OR 1.28, 95\% Cl 1.09-1.50; $\mathrm{p}=0.002$ ), and the LUTS-depression association increased as a function of the number of ALES $(F=22.62 ; b=0.061 ; p<0.001)$. C-reactive protein was found to facilitate the association between LUTS and depression, suggesting activation of the central inflammatory signaling as a biological pathway underlying the association. These results serve as a caution that men with LUTS and a history of ALEs may be vulnerable to depressive symptoms.

\section{References}

1. Drake MJ, Chapple C, Esen AA, et al. BESIDE study investigators. Efficacy and safety of mirabegron add-on therapy to solifenacin in incontinent overactive bladder patients with an inadequate response to initial 4-week solifenacin monotherapy: A randomized, double-blind multicentre phase 3B study (BESIDE). Eur Urol 2016 [Epub before print].

2. Drake MJ, Chappie C, Esen AA, et al. Cardiovascular (CV) assessments in a randomized, double-blind, phase $3 \mathrm{~b}$ trial of mirabegron add-on treatment in incontinent overactive bladder (OAB) patients with an inadequate response to solifenacin monotherapy. Eur Urol Supp/ 2016;15(suppl 3):e872. http://dx.doi. org/10.1016/S1569-9056(16)60874-6

3. Coelho A, Gillespie J, Cruz F. Beta-3 adrenoceptor expression in the human urinary bladder nerve fibers. Eur Urol Suppl 2016;15(suppl 3):e308. http://dx.doi.org/10.1016/S1569-9056(16)60310-X

4. Everaert K, Sriram R, Kohan A, et al. The efficacy and safety of onabotulinumtoxinA and solifenacin compared to placebo in solifenacin-naïve patients with idiopathic overactive bladder: Results from a multicentre, randomized, double-blind, phase 3b trial. Eur Urol Suppl 2016;15(suppl 3):e877. http:// dx.doi.org/10.1016/S1569-9056(16)60879-5

5. Yoshida M, Gotoh M, Kageyama $S$, et al. Mirabegron improves nocturia and nocturia associated QoL and sleep quality. Eur Urol Suppl 2016;15(suppl 3):e538. http://dx.doi.org/10.1016/S1569$9056(16) 60540-7$

6. Cho KJ, Lee ZZ, Lee JG, et al. Efficacy and safety of desmopression "add-on" therapy in men with persistent nocturia under alpha blocker monotherapy for LUTS: A randomized, double-blind, placebo-controlled study. Eur Urol Supp/ 2016;15(suppl 3):e543. http://dx.doi.org/10.1016/S1569-9056(16)60545-6

7. Roehrborn CG, Rukstalis DB, Barkin J, et al. Three year results of the prostatic urethral L.I.F.T. study. Can J Urol 2015;22:7772-82.

8. Roehrborn C, Gange S, Shore N, et al. Four year results from the largest, prospective, randomized study of prostatic urethral lift (PUL). Eur Urol Suppl 2016;15(suppl 3):e1077. http://dx.doi.org/10.1016/ S1569-9056(16)61078-3

9. Abdelmoteleb H, Hashim H. Association between free flow and bladder outlet obstruction index and bladder contractility index. Eur Urol Suppl 2016;15(suppl 3):e987. http://dx.doi.org/10.1016/ S1569-9056(16)60988-0

10. Gacci $M$, Drake $M$, Castellini $G$, et al. Lower urinary tract symptoms, depression and adverse life events: Data from the European Male Ageing Study. Eur Urol Suppl 2016;15(suppl 3):e98. http://dx.doi. org/10.1016/S1569-9056(16)60100-8 